\title{
Deferasirox for transfusion-dependent patients with myelodysplastic syndromes: safety, efficacy, and beyond (GIMEMA MDS0306 Trial)
}

Emanuele Angelucci ${ }^{1}$, Valeria Santini ${ }^{2}$, Anna Angela Di Tucci ${ }^{1}$, Giulia Quaresmini ${ }^{3}$, Carlo Finelli ${ }^{4}$, Antonio Volpe ${ }^{5}$, Giovanni Quarta ${ }^{6}$, Flavia Rivellini ${ }^{7}$, Grazia Sanpaolo ${ }^{8}$, Daniela Cilloni ${ }^{9}$, Flavia Salvi $^{10}$, Giovanni Caocci ${ }^{11}$, Alfredo Molteni ${ }^{12}$, Daniele Vallisa ${ }^{13}$, Maria Teresa Voso ${ }^{14}$, Susanna Fenu ${ }^{15}$, Lorenza Borin $^{16}$, Giancarlo Latte ${ }^{17}$, Giuliana Alimena ${ }^{18}$, Sergio Storti ${ }^{19}$, Alfonso Piciocchi ${ }^{20}$, Paola Fazi ${ }^{20}$, Marco Vignetti ${ }^{20}$, Sante Tura ${ }^{21}$

${ }^{1}$ Hematology and Bone Marrow Transplantation Unit, Ospedale Oncologico di Riferimento Regionale "Armando Businco", Cagliari; ${ }^{2}$ Division of Hematology, University of Florence, Florence; ${ }^{3}$ Hematology and Bone Marrow Transplant Unit, Azienda Ospedaliera Papa Giovanni XXIII, Bergamo; ${ }^{4}$ Division of Hematology, Policlinico S. Orsola-Malpighi, Bologna; ${ }^{5}$ Division of Hematology, San Giuseppe Moscati Hospital, Avellino; ${ }^{6}$ Division of Hematology, Ospedale A. Perrino, Brindisi; ${ }^{7}$ UO Medicina Interna e Onco-Ematologica P.O. "Umberto I", Nocera Inferiore; ${ }^{8}$ Division of Hematology, IRCCS "Casa Sollievo della Sofferenza", San Giovanni Rotondo; ${ }^{9}$ Department of Clinical and Biological Sciences, University of Turin, Turin; ${ }^{10}$ Division of Hematology, A.O. Nazionale Santi Antonio e Biagio e C. Arrigo, Alessandria; ${ }^{11}$ Bone Marrow Transplantation Center, R. Binaghi Hospital, Cagliari; ${ }^{12}$ Division of Hematology, Niguarda Ca' Granda Hospital, Milan; ${ }^{13}$ Division of Medical Oncology and Hematology, Hospital of Piacenza, Piacenza; ${ }^{14}$ Division of Hematology, Università Cattolica del Sacro Cuore, Rome; ${ }^{15}$ Division of Hematology, San Giovanni Hospital, Rome; ${ }^{16}$ Division of Hematology, Monza; ${ }^{17}$ Division of Hematology, S. Francesco Hospital, Nuoro; ${ }^{18}$ Division of Hematology, "Sapienza" University of Rome, Rome; ${ }^{19}$ Division of Hematology and Medical Oncology, Campobasso University, Campobasso; ${ }^{20}$ GIMEMA Data Center, GIMEMA Foundation, Rome; ${ }^{21}$ University of Bologna, Bologna, Italy

\begin{abstract}
Background: In the absence of randomized, controlled trial data to support iron chelation therapy in transfusion-dependent patients with myelodysplastic syndromes (MDS), continued evidence from large prospective clinical trials evaluating the efficacy and safety of iron chelation therapy in this patient population is warranted. Methods: The safety and efficacy of deferasirox was examined in a prospective, open-label, singlearm, multicenter trial of transfusion-dependent patients with International Prognostic Scoring System low- or intermediate-1-risk MDS and evidence of transfusion-related iron overload. The effects of deferasirox therapy on hematological response and disease progression were also examined. Results: Of 159 participants enrolled from 37 Italian centers, 152 received $\geq 1$ dose of deferasirox (initiated at 10-20 mg/kg/day and titrated as appropriate), and 68 completed the study. Of 84 patients who discontinued deferasirox therapy, 22 died during the trial, and 28 withdrew due to an adverse event (AE). Fourteen treatment-related grade 3 AEs occurred in 11 patients, whereas no grade 4 or 5 drug-related AEs were reported. Significant risks for dropout were a higher serum ferritin level at baseline, a higher MDS-Specific Comorbidity Index, and a shorter diagnosis-enrollment interval. Median serum ferritin level fell from $1966 \mathrm{ng} / \mathrm{mL}$ to $1475 \mathrm{ng} / \mathrm{mL}(P<0.0001)$. The cumulative incidence of transfusion independence, adjusted for death and disease progression, was $2.6 \%, 12.3 \%$, and $15.5 \%$ after 6,9 , and 12 months, respectively. Conclusions: Deferasirox therapy in transfusion-dependent patients with MDS was moderately well tolerated and effectively lowered serum ferritin levels. Positive hematological responses were observed, and a subset of patients achieved transfusion independence.
\end{abstract}

Key words deferasirox; chelation therapy; myelodysplastic syndromes; iron chelation; iron overload; serum ferritin; safety

Correspondence Emanuele Angelucci, MD, Hematology and Bone Marrow Transplantation Unit, Ospedale Oncologico di Riferimento Regionale "Armando Businco", Via Edward Jenner Cagliari, 09121 Italy. Tel: +39 0706092061 ; Fax: +39 070 6092065;

e-mail: emnang@tin.it

Presented at: Oral Presentation at 54th American Society of Hematology annual meeting (Atlanta 2012). 
Myelodysplastic syndromes (MDS) represent a heterogeneous group of hematological disorders characterized by dysplastic and ineffective blood cell production. Most patients will present with anemia and its associated symptoms and go on to require blood transfusions. A substantial number will develop transfusion dependence, which is associated with poorer outcomes presumed, in part, to be a consequence of transfusional siderosis (1). Based on this understanding and the impressive benefits seen in thalassemia populations, the US Food and Drug Administration (FDA) and the European Medicines Agency (EMA) approved the oral iron chelator deferasirox in 2005 for the treatment of 'chronic iron overload due to blood transfusions', inclusive of transfusion-dependent patients with MDS. Since its approval, the body of evidence on the efficacy and safety of deferasirox in patients with MDS has been slowly expanding (2-9), although data from randomized, controlled clinical trials are still lacking (10). Until such data becomes available, further evidence from prospective trials evaluating and confirming the efficacy and safety of deferasirox therapy in MDS remains essential; especially considering the advanced age and high prevalence of comorbidity in this patient population compared with other congenital or acquired anemias. Hematologic responses have also been observed in patients with MDS receiving deferasirox; although evidence mostly comes from case series or observational studies, only few clinical trials provided such data through exploratory or post hoc analyses $(2,3,11)$. There is contention in the hematological community as to the degree to which iron is responsible for deleterious outcomes in transfusion-dependent patients with MDS and to the evidence base from which iron chelation guideline recommendations have been made. There is, however, agreement regarding the need for well-organized, prospective data to examine the use of this potentially efficacious therapeutic tool in MDS populations (12-15).

With this background, the purpose of this trial was to establish the safety and efficacy of deferasirox in a realworld population of transfusion-dependent patients with MDS and to evaluate its role in achieving hematologic responses and transfusion independence.

\section{Methods}

\section{Participants}

GIMEMA MDS0306 was a prospective, open-label, singlearm, Italian multicenter study of deferasirox in transfusiondependent patients with MDS (Appendix 1). Enrollment of participants began in July 2007 and concluded in February 2010. Eligible patients were as follows: $18 \mathrm{yr}$ or older with MDS; International Prognostic Scoring System (IPSS) (16) low- or intermediate-1-risk; diagnosed with transfusion dependency, defined as requiring $\geq 1$ unit of packed red blood cells (pRBC) in $\leq 8 \mathrm{wk}$ averaged over 4 months before the start of treatment; diagnosed with transfusional siderosis following a minimum of 20 units of pRBC transfusions; and with a serum ferritin level of $\geq 1000 \mathrm{ng} / \mathrm{mL}$. The main exclusion criteria were as follows: an estimated creatinine clearance $<60 \mathrm{~mL} / \mathrm{min}$ at screening; aspartate aminotransferase (AST)/alanine aminotransferase (ALT) $>500 \mathrm{U} / \mathrm{L}$; active hepatitis $\mathrm{B}$ or $\mathrm{C}$ infections; ongoing treatment with chelation therapy; previous treatment with thalidomide or its analogs, azacitidine or arsenic in the 4 wk preceding screening (although these drugs were permitted during the study, if clinically indicated); uncontrolled hypertension or cardiac disease; and Eastern Cooperative Oncology Group (ECOG) (17) performance status (PS) $\geq 2$. All patients provided written informed consent. The study protocol was approved by ethical committees and institutional review boards of all the participating centers and was conducted in accordance with the Declaration of Helsinki. This trial is registered at www. clinicaltrials.gov as \#NCT00469560. All authors had access to primary clinical trial data for analysis.

\section{Interventions and outcomes}

In this open-label, single-arm trial, patients were scheduled to receive daily oral deferasirox at a dose between 10 and $30 \mathrm{mg} / \mathrm{kg}$ of body weight for $1 \mathrm{yr}$. In patients receiving $<7 \mathrm{~mL} / \mathrm{kg}$ of pRBC (approximately $<2$ units per month), a lower deferasirox dose of $10 \mathrm{mg} / \mathrm{kg} / \mathrm{d}$ was considered. The primary study outcome was safety and tolerability as determined by Common Terminology Criteria for Adverse Events (CTCAE) version 3 (18). Adverse events (AEs) were recorded at individual participating centers and categorized according to their relationship with the trial drug as follows: certainly related, probably related, possibly related, not likely related, or not related. Events categorized as certainly, probably or possibly related to the trial drug were considered treatment-related. Secondary study outcomes were efficacy as determined by changes in serum ferritin level from baseline, transfusion requirement and hematological response defined by the International Working Group (IWG) 2006 criteria (19), disease progression defined according to World Health Organization (WHO) classification criteria (20), and quality of life (QOL). Erythroid response was defined as achievement of transfusion independence. Transfusion independence was defined as freedom from transfusion for three consecutive months [interval periods were modified from initial study design to 3 months, in accordance with new consensus guidelines released during the study period (21)]. Disease progression was defined as advance to intermediate- 2 or high IPSS, or development of acute leukemia. Primary and secondary outcomes were assessed at 1-month intervals until study completion. QOL outcomes will be reported in a separate report. 


\section{Statistical analysis}

The primary end point of the study was the rate of treatment-related AEs ( $\geq$ grade 3 ). Assuming a $2 \%$ primary end point event rate, power calculations showed that 150 patients would provide $87 \%$ power with a significance level of 0.05 . Descriptive statistics were used to report trial population demographics and observed toxicity. All reported symptoms and AEs were coded according to the Medical Dictionary for Regulatory Activities (MedDRA) System Organ Class categorization version 15.1 [International Federation of Pharmaceutical Manufacturers and Associations (IFPMA), Geneva, Switzerland]. Bivariate comparisons between study variables were carried out using the chi-square or Fisher's exact test and the Wilcoxon test. The 12-month cumulative incidence of dropout and transfusion independence were estimated using the appropriate non-parametric method considering death and disease progression as competitive risks. Cumulative incidence was estimated using the cmprsk package for R, version 2.15 (R Foundation for Statistical Computing, Vienna, Austria). To determine the effect of baseline predictors on dropout rates and transfusion independence, Cox regression with the Fine-Gray method was used (22). The variables considered for multivariate analysis were age, IPSS risk group, diagnosis-recruitment interval, number of blood transfusions received prior to treatment with the trial drug, duration of transfusion dependence, and cytogenetic factors.

With the exception of hematological responses, analyses were performed on a treatment-received basis, and participants who withdrew before receiving the trial drug were excluded. Hematological response rates are reported as an intent-to-treat analysis. Enrolled patients who withdrew from treatment before a response was reported were counted as non-responders. For hematological response analysis, patients receiving other hematological modifying drugs were excluded to determine the effect of the trial drug. Patients eligible for platelets response analysis had a platelet count of $<100 \times 10^{9} / \mathrm{L}$ at baseline, whereas those considered for a neutrophil response had a neutrophil count $<1 \times 10^{9} / \mathrm{L}$. A platelet response was defined as either an increase of $\geq 30 \times 10^{9} / \mathrm{L}$ (in patients with $\geq 20$ at screening) or an increase to $\geq 20 \times 10^{9} / \mathrm{L}$ (in patients with screening values $<20$ ) for three consecutive readings. A neutrophil response was defined as a $100 \%$ increase in neutrophil count in conjunction with an absolute increase $>0.5 \times 10^{9} / \mathrm{L}$ on three consecutive readings. In accordance with our criteria for transfusion independence, platelet and neutrophil responses were assessed over a minimum interval of 3 months (21). Friedman's test was used to examine differences in hematological parameters. If a significant variation was observed, values recorded over two consecutive months were pooled for comparison. Differences between responders and nonresponders were evaluated with the Wilcoxon rank test.
In all, 95\% confidence intervals (CI) were reported for main summary statistics, and all statistical comparisons were based on two-tailed tests with a 5\% significance level. Statistical analyses were performed by SAS Version 9.2 statistical software (SAS Institute, Cary, NC, USA).

\section{Results}

The characteristics of the study population were typical for patients with MDS; Median age was $72 \mathrm{yr}$ (IQR 66-77), and $96(63 \%)$ of participants were men. The median duration of disease at enrollment was 32 months (IQR 17-54), and median number of units of pRBCs received was 37 (IQR 22-63). Both the median Cumulative Illness Rating Scale (CIRS) (23) and Charlson (24) comorbidity scores were 0 (IQR 0-1), and the median CIRS severity score was 0.2 (IQR 0-1). The MDS-Specific Comorbidity Index (25) was calculated retrospectively, and median risk was low (IQR low-intermediate). Hematological parameters at baseline are described in Table 1.

Table 1 Baseline characteristics at enrollment for patients that proceeded to treatment

\begin{tabular}{|c|c|}
\hline Characteristic & $n=152$ \\
\hline Age $(y r)$ & 72 (IQR 66-77, range 24-87) \\
\hline \multicolumn{2}{|l|}{ Sex, $n(\%)$} \\
\hline Male & $96(63.2)$ \\
\hline Female & $56(36.8)$ \\
\hline \multicolumn{2}{|l|}{ IPSS score, $n(\%)$} \\
\hline Low & $61(40.7)$ \\
\hline Intermediate & $89(59.3)$ \\
\hline CIRS comorbidity index & 0 (IQR 0-1, range $0-2)$ \\
\hline CIRS severity index & 0.2 (IQR 0.1-0.5, range 0-5) \\
\hline \multicolumn{2}{|c|}{ MDS-Specific Comorbidity Index, $n(\%)$} \\
\hline Low & $111(74.0)$ \\
\hline Intermediate & $33(22.0)$ \\
\hline High & $6(4.0)$ \\
\hline $\begin{array}{l}\text { Time since initial MDS } \\
\text { diagnosis (months) }\end{array}$ & 32 (IQR 17-54, range 3-204) \\
\hline $\begin{array}{l}\text { Time since starting } \\
\text { transfusion (months) }\end{array}$ & 21 (IQR 10-36, range 2-120) \\
\hline $\begin{array}{l}\text { Units of packed red } \\
\text { cells transfused }\end{array}$ & 37 (IQR 22-63, range 20-420) \\
\hline $\begin{array}{l}\text { Pretransfusion } \\
\text { hemoglobin }(\mathrm{g} / \mathrm{dL}) \\
\text { (mean } \pm \mathrm{SD})\end{array}$ & $8.3 \pm 0.9$ \\
\hline Serum ferritin (ng/mL) & $\begin{array}{r}1966 \text { (IOR 1416-2998, } \\
\text { range 709-13395) }\end{array}$ \\
\hline \multicolumn{2}{|c|}{$\begin{array}{l}\text { Values are median (IQR) unless otherwise indicated. IPSS indicates } \\
\text { International Prognostic Scoring System; CIRS, cumulative illness rat- } \\
\text { ing scale; and MDS, myelodysplastic syndrome. Numbers may not } \\
\text { equal sum of study population ( } n=152) \text { due to missing data. Data } \\
\text { were missing with respect to IPSS group ( } n=2) \text {, CIRS comorbidity } \\
\text { index ( } n=3) \text {, CIRS severity index ( } n=2) \text {, and MDS-Specific Comor- } \\
\text { bidity Index }(n=2) \text {. }\end{array}$} \\
\hline
\end{tabular}




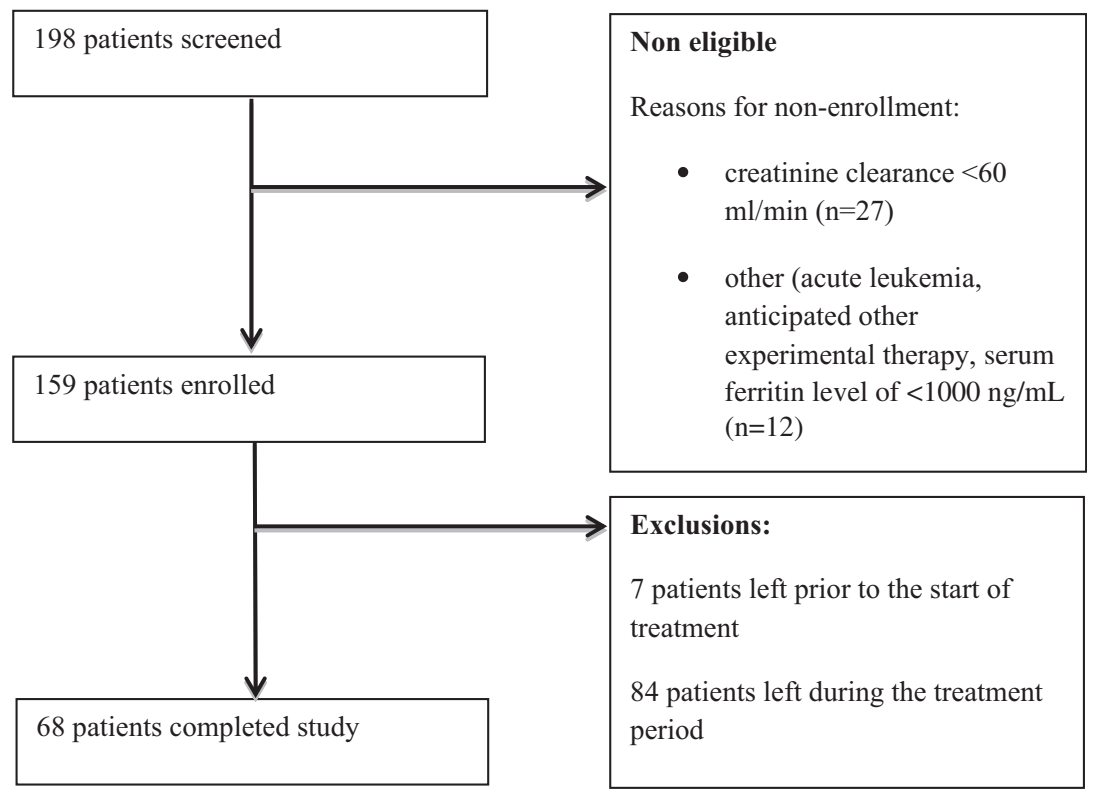

Figure 1 Consolidated Standards on Reporting Trials (CONSORT) diagram of the study population.

Of 159 patients enrolled at 37 Italian centers, 152 (96\%) patients received at least one dose of trial drug, and 68 (43\%) completed the trial (Fig. 1). Seven enrolled patients did not start treatment with the trial drug due to death $(n=2)$, AEs $(n=2)$, withdrawal of consent $(n=1)$, loss to follow-up $(n=1)$, and ineligibility $(n=1)$. The daily median dose of deferasirox was $10 \mathrm{mg} / \mathrm{kg}$ (IQR 6.2-14) in the first month and $15.0 \mathrm{mg} / \mathrm{kg}$ (IQR 9.6-19.5) for the remainder of the trial. No significant differences were observed in trial drug doses between months 2 and $12(P=0.93)$. Among 84 patients who discontinued deferasirox therapy (Table 2), 28 (33\%) withdrew due to a drug-related or unrelated AEs (Fig. S1, Table S1), and 22 (26\%) died during the trial period (Table S2). The cumulative incidence of dropout, adjusted for death and disease progression, was 19\% and $27 \%$ at 6 and 12 months, respectively. Cumulative incidence of disease progression or death was $13 \%$ and $20 \%$ at 6 and 12 months, respectively. Both a higher baseline serum ferritin level (HR 1.02, 95\% CI 1.01-1.04) and a higher MDSSpecific Comorbidity Index (HR 1.42, 95\% CI 1.13-1.78) were significant predictors of trial dropout in multivariate

Table 2 Reasons for discontinuation of deferasirox therapy

\begin{tabular}{lc}
\hline Cause & $n(\%)$ \\
\hline Adverse event & $28(33.3)$ \\
Death & $22(26.2)$ \\
Disease progression & $8(9.5)$ \\
Withdrawal of consent & $9(10.7)$ \\
Lost at follow-up & $8(9.5)$ \\
No response & $2(2.4)$ \\
Serum ferritin $<500 \mathrm{ng} / \mathrm{mL}$ & $2(2.4)$ \\
Medical decision & $5(6.0)$ \\
Total & $84(100)$ \\
\hline
\end{tabular}

analysis, whereas a longer diagnosis-enrollment interval (duration of disease) was associated with a reduced dropout rate (HR 0.98, 95\% CI 0.97-0.99) (Table 3). While a higher CIRS comorbidity index (HR 1.74, 95\% CI 0.96-3.17) and a higher drug dose (HR 0.95, 95\% CI 0.90-0.99) demonstrated an association with trial dropout on univariate analysis, these variables did not remain as significant predictors of dropout in multivariate analysis.

\section{Safety and tolerability}

Among 303 AEs recorded in 107 patients, 92 (30\%) events were treatment-related, including 14 grade 3 events in 11 patients (Table 4). No treatment-related grade 4 or 5 events were recorded. Of the treatment-related AEs, 42 (45\%) were gastrointestinal, $15(16 \%)$ were renal or urinary disorders, and $5(5 \%)$ were hepatobiliary (Fig. S1 and Table S1). No grade 4 or 5 renal AEs occurred in either treatment-related or nonrelated categories. There were 30 grade 4 or 5 events among the 211 non-related AEs (Table S2). The most common grade 4 AEs were thrombocytopenia (three events) and congestive cardiac failure (two events). Of 22 deaths that occurred during the study period, respiratory disorders were the cause of death in six patients $(32 \%)$ in addition to two deaths from pneumonia, central nervous system hemorrhages were the cause of death in four patients (18\%), and cardiac arrest or failure were the cause of death in three patients $(14 \%)$.

\section{Efficacy}

Median serum ferritin level at baseline was $1966 \mathrm{ng} / \mathrm{mL}$ (IQR 1413-2907) compared with $1476 \mathrm{ng} / \mathrm{mL}$ (IQR 9152010) at trial completion $(P<0.001)$ (Fig. S2). In conjunction 
Table 3 Univariate and multivariate analysis of risk factors for study dropout using the Fine -Gray model

\begin{tabular}{|c|c|c|c|c|c|c|}
\hline \multirow[b]{2}{*}{ Variable } & \multicolumn{3}{|c|}{ Univariate analysis } & \multicolumn{3}{|c|}{ Multivariate analysis } \\
\hline & $\mathrm{HR}$ & $(95 \% \mathrm{Cl})$ & $P$ & $\mathrm{HR}$ & $(95 \% \mathrm{Cl})$ & $P$ \\
\hline $\begin{array}{l}\text { Duration of } \\
\text { disease } \\
\text { (months) }\end{array}$ & 0.99 & $(0.98-1.00)$ & 0.007 & 0.98 & $(0.97-0.99)$ & 0.004 \\
\hline $\begin{array}{l}\text { Serum } \\
\text { ferritin } \\
\text { (ng/mL) }\end{array}$ & 1.02 & $(1.01-1.04)$ & 0.004 & 1.02 & $(1.01-1.04)$ & 0.002 \\
\hline CIRS & 1.74 & $(0.96-3.17)$ & 0.07 & - & - & - \\
\hline MDS-Cl & 1.45 & $(1.14-1.84)$ & 0.003 & 1.42 & $(1.13-1.78)$ & 0.003 \\
\hline $\begin{array}{l}\text { Deferasirox } \\
\text { dose } \\
(\mathrm{mg} / \mathrm{kg} / \mathrm{d})\end{array}$ & 0.95 & $(0.90-0.99)$ & 0.02 & - & - & - \\
\hline
\end{tabular}

CIRS, Cumulative IIIness Rating Scale; MDS-Cl, Myelodysplastic Syndrome-Specific Comorbidity Index.

Table 4 Adverse events by severity grade, causal likelihood, and organ system

\begin{tabular}{|c|c|c|c|c|}
\hline Relationship & Events & \multicolumn{3}{|c|}{ Patients* $n(\%)$} \\
\hline \multicolumn{5}{|c|}{ Treatment-related } \\
\hline Grade 1 & 38 & \multicolumn{3}{|c|}{$24(15.8)$} \\
\hline Grade 2 & 40 & \multicolumn{3}{|c|}{$29(19.1)$} \\
\hline Grade 3 & 14 & \multicolumn{3}{|c|}{$11(7.2)$} \\
\hline \multicolumn{5}{|l|}{ Non-related } \\
\hline Grade 1 & 71 & \multicolumn{3}{|c|}{$36(23.7)$} \\
\hline Grade 2 & 70 & \multicolumn{3}{|c|}{$41(27.0)$} \\
\hline Grade 3 & 40 & \multicolumn{3}{|c|}{$25(16.4)$} \\
\hline Grade 4 & 8 & \multicolumn{3}{|c|}{$6(3.9)$} \\
\hline Grade 5 & 22 & \multicolumn{3}{|c|}{$22(13.8)$} \\
\hline Total & 303 & \multicolumn{3}{|c|}{$107(70.4)$} \\
\hline \multirow{2}{*}{\multicolumn{2}{|c|}{$\begin{array}{l}\text { Organ system } \\
\text { Grade }\end{array}$}} & \multicolumn{3}{|c|}{$\begin{array}{l}\text { Treatment- } \\
\text { related events }\end{array}$} \\
\hline & & & 2 & 3 \\
\hline \multicolumn{2}{|l|}{ Cardiac } & - & 1 & \\
\hline \multicolumn{2}{|l|}{ Eye } & 1 & - & - \\
\hline \multicolumn{2}{|l|}{ Gastrointestinal } & 17 & 19 & 6 \\
\hline \multicolumn{2}{|c|}{ General disorders and administration site conditions } & - & - & 1 \\
\hline \multicolumn{2}{|l|}{ Hepato-biliary } & - & 3 & 2 \\
\hline \multicolumn{2}{|l|}{ Investigations } & 8 & 6 & - \\
\hline \multicolumn{2}{|c|}{ Metabolism and nutrition } & - & 1 & 2 \\
\hline \multicolumn{2}{|c|}{ Musculoskeletal and connective tissue } & 1 & 3 & - \\
\hline \multicolumn{2}{|c|}{ Renal and urinary } & 10 & 4 & 1 \\
\hline \multicolumn{2}{|c|}{ Skin and subcutaneous tissue } & 1 & 3 & 2 \\
\hline \multicolumn{2}{|l|}{ Total } & 38 & 40 & 14 \\
\hline
\end{tabular}

* Patients may be listed multiple times in case of multiple adverse events, and therefore, total counts of adverse events do not correspond with patient numbers.

with a fall in median serum ferritin level, there were overall reductions in median ALT and AST levels during the study period (Fig. S3). Changes in median serum ferritin level correlated positively with AST levels between months 1 and $11(P<0.001)$ and with ALT levels, all throughout the follow-up period $(P<0.01)$. Median creatinine values increased from $0.87 \mathrm{mg} / \mathrm{dL}$ (IQR $0.42-1.32$ ) at baseline to $0.98 \mathrm{mg} / \mathrm{dL}$ (IQR $0.58-1.40$ ) at end of study (Fig. S4).

\section{Hematological response and transfusion independence}

An erythroid, neutrophil, and platelet response was observed in $11 \%(16 / 145), 3 \%(4 / 125)$, and $15 \%(19 / 125)$ of patients, respectively. Serum ferritin levels were significantly lower among patients with a platelet response than counterparts without a platelet response at month 4 of follow-up $(P=0.01)$ and non-significantly lower between months 1 and 10 .

The cumulative frequency of patients achieving transfusion independence was three patients at 3 months, 13 patients at 9 months, and 16 patients at 12 months. Six patients with an erythroid response who were receiving concomitant MDS medications (rHuEpo, 5 Azacitidine and lenalidomide) were excluded from these frequencies; however, 24 patients who received these medications, but did not present any hematological response, were included in the denominator for calculations. The cumulative incidence of transfusion independence adjusted for death and disease progression was estimated to be $2.6 \%, 12.3 \%$, and $15.5 \%$ at 6 , 9 , and 12 months, respectively (Fig. S5). The median hemoglobin level during transfusion independence was $8.1 \mathrm{~g} / \mathrm{dL}$ (IQR 7.5-8.5). There were no significant differences in median pretransfusional hemoglobin levels during the study period $(P=0.27)$. The median number of $\mathrm{pRBC}$ transfusions received per month was 3 (IQR 2-5) at baseline compared with 1 (IQR $0-4)$ at 12 months $(P<0.001$, Fig. 2). When stratified for the number of $\mathrm{pRBC}$ transfusions received per month at baseline, patients requiring $>3$ pRBC units experienced the greatest reduction in transfusion frequency; median transfusion requirement in this sub-cohort fell from 5 to 2 pRBC units per month. Statistically significant reductions in $\mathrm{pRBC}$ transfusion requirements were confirmed in 'per protocol' analyses (data not shown).

Among individuals who achieved transfusion independence, there was no significant reduction in median serum ferritin level compared with those who remained transfusion dependent. However, those who achieved transfusion independence had a lower, non-statistically significant, median serum ferritin level at baseline when compared with patients who remained transfusion dependent (1598 ng/mL versus $1902 \mathrm{ng} / \mathrm{mL}$ ). We observed no association between transfusion independence and either a platelet or neutrophil response. No patients became transfusion independent in combination with a platelet and neutrophil response. Interestingly, we identified no significant predictors of transfusion independence in univariate analysis that considered age, IPSS risk status, MDS-CI, diagnosis-enrollment interval, number of previous pRBC 


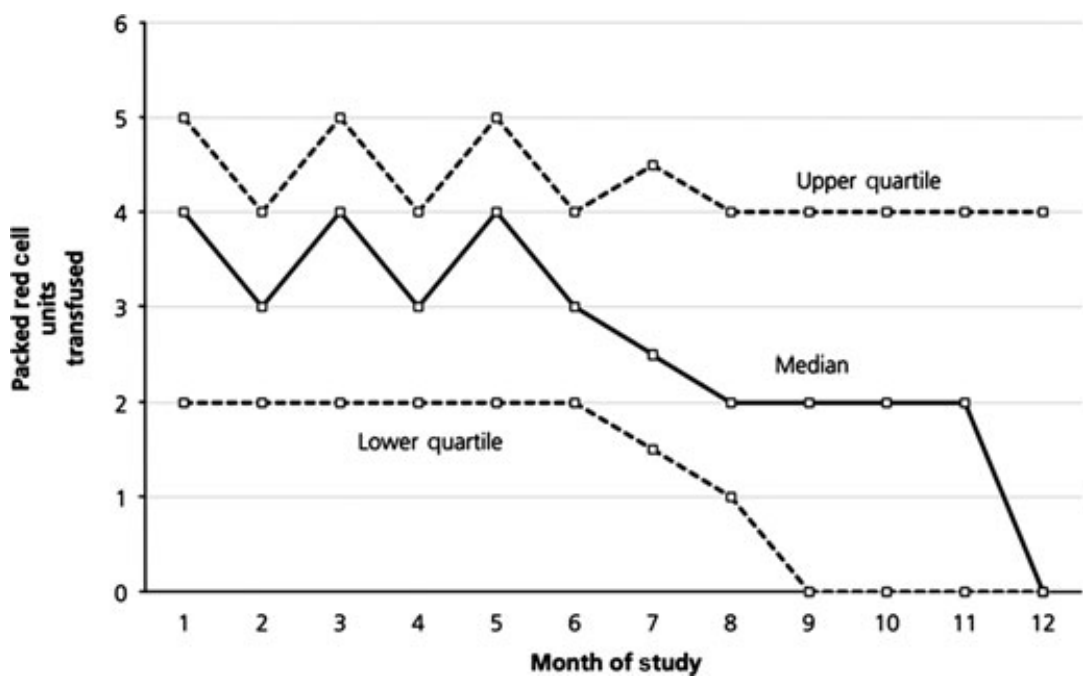

Figure 2 Packed red blood cell transfusion requirements per month. Patients receiving concurrent MDS medication (rHuEpo, 5-Azacitidine, Lenalidomide) were excluded, $P<0.0001$.

transfusions, duration of transfusion dependence, baseline serum ferritin level, and cytogenetic factors.

\section{Discussion}

In this multicenter prospective trial, we demonstrated the safety and efficacy of deferasirox chelation therapy for lower risk, transfusion-dependent patients with MDS. We provided prospective evidence for hematological benefit in respect of achieving transfusional independence in association with deferasirox therapy. Further, we provided indirect supporting evidence for alleviating end-organ damage, as demonstrated by improvements in transaminase levels (albeit modest over the short-term), which may reflect decreased hepatocellular injury.

Supportive measures and pRBC transfusion form the mainstay of management for patients with MDS in low- and intermediate-1-risk groups, and transfusion dependence is common. Retrospective studies have confirmed a negative association between transfusion dependency and overall survival in patients with MDS(26-28). Examination of US registry data also found associations between transfusion dependence and cardiac, liver, and metabolic disease, which are classically seen in iron-overload syndromes (29). Our study remains the largest to provide prospective data on hematologic responses as a study endpoint in patients with MDS receiving deferasirox, with a dedicated definition for transfusion independence. The erythroid response observed in our study consisted primarily of a transfusion rather than a hemoglobin response. Among 145 patients included for response analysis (by intention to treat), 16 (11\%) patients became transfusion independent. In a recent prematurely closed study by Nolte and colleagues (2), only two of 33
(6\%) patients achieved an erythroid response following deferasirox treatment for 12 months. In post hoc analysis from a larger open-label, single-arm, 3 -yr phase 2 trial in the US and Canada (3), erythroid responses were observed in $15 \%$ of patients (26/173 patients). Of note, three of these patients were also on other MDS therapies (one on lenalidomide and two on erythropoietin). Post hoc analysis from the EPIC trial (prospective, 1-yr, multicenter, open-label, phase $3 \mathrm{~b}$ trial) (11) also showed a $21.5 \%$ (53/247 patients) rate of erythroid response upon deferasirox therapy, with $11.3 \%$ of patients having a transfusion-only erythroid response and $8.9 \%$ of patients having a hemoglobin-only erythroid response. Differences in response rates between studies may be attributed to (i) dose of deferasirox (lower in our study in response to non-severe AEs), (ii) duration of therapy and observation, (iii) inclusion/exclusion or patients receiving other erythroid stimulants from the analysis, and (iv) pretreatment hemoglobin level and transfusion requirements. As noted earlier, we observed a significant reduction in transfusion frequency during the trial period even in the absence of significant increase in hemoglobin levels. These findings may, in part, reflect symptomatic improvements independent of hemoglobin levels, as our transfusion policy was consistent throughout the study period. We observed a trend, albeit statistically non-significant, for increased improvement in pRBC transfusion requirement in patients with higher transfusional need, perhaps indicating that a greater benefit is achieved when there is a particularly high level of oxidative stress. Collectively, these findings provide the best current evidence for the possibility of developing a hematological response to deferasirox therapy. Importantly, patients receiving other hematological modifying drugs were excluded from hematological response analyses. 
We did not identify any remarkable relationship between iron status or depletion and transfusion independence in our study, which is in agreement to previous reports showing no association between changes in iron indices and erythroid responses $(3,11)$. This may indicate that the effects of deferasirox on the bone marrow environment extend beyond those of iron depletion or scavenging reactive oxygen species $(30,31)$. Nuclear factor-kappaB inhibition by deferasirox is one such effect, which is not seen from other chelators and is iron and reactive oxygen species scavenging independent (32). Deferasirox may also be interfering with the dynamic regulation between erythropoiesis and the hepatic peptide hepcidin, as deferasirox treatment has been shown to increase hepcidin levels (33). A similar observation was recently made in thalassemia patients, where the rate of mobilization of hematopoietic peripheral progenitors was higher with deferasirox than other chelators and was independent of iron depletion (34).

We observed a reduction in serum ferritin level of more than $36 \%$ following 12 months of deferasirox therapy. This is comparable to data from recent clinical trials documenting reductions of $20-40 \%(2,3,5,6)$. There was a significant correlation between decline in serum ferritin and improvement in hepatic transaminases, confirming previous observations in chelated patients with $\operatorname{MDS}(3,6)$. Serum ferritin level has been identified as a poor prognostic indicator in patients with MDS. In a Japanese cohort of 292 patients with MDS and aplastic anemia, 37 of 38 patients who died of hepatic or cardiac failure had serum ferritin levels $>1000 \mathrm{ng} / \mathrm{mL}$ (35). These findings led to the hypothesis that iron overload may be responsible for poorer outcome in patients with MDS and justifies the relationship between transfusion dependence and increased morbidity or mortality risk, although a confounding effect of underlying anemia cannot be fully dismissed. Irrespectively, data from several retrospective cohorts observed improved survival in patients with MDS receiving chelation therapy $(36,37)$.

In our study, $45 \%$ of patients who began deferasirox therapy completed treatment, a figure comparable with that of previous reports $(3,6)$. Poor trial completion rates with patients with MDS have been compounded by difficulties in compliance, and this likely reflects the elderly and frail population with poor motivation with regards to their disease and the potential benefits of iron chelation therapy. Interestingly, duration of disease was associated with a higher likelihood of trial completion in our study; potentially reflecting improved patient education and understanding of the disease process. The advanced age and poor health of patients with MDS may explain the high incidence of non-treatmentrelated AEs reported in this cohort. Indeed, a greater MDSCI was associated with a higher dropout rate in the present study. We observed no treatment-related deaths, doselimiting toxic effects, or treatment-related grade 4 AEs, and the majority of treatment-related AEs were minor. Of these, most were easily managed gastrointestinal symptoms, although gastrointestinal disturbances have been recognized as the main cause for discontinuation of deferasirox treatment in reports to date (38). A large minority (42\%) of patients in the current study experienced treatment-related AEs compared with $66 \%$ in the 1-yr EPIC study (6). Notably, renal AEs were lower than previously reported, with only eight treatment-related events of increased serum creatinine and one event of renal failure observed. The reasons for this are unclear but may relate to improved management, with effective monitoring and appropriate dose titration.

There are several limitations to this study that warrant consideration. Similar to other available studies, our study lacked a control arm to better confirm the efficacy of deferasirox therapy. It is more challenging to confirm that hematological responses or the development of transfusion independence is directly related to deferasirox therapy in the absence of a placebo control. Observational reports suggest that spontaneous improvement in hematological parameters and the development of transfusion independence can occur off treatment (39), although such instances are rare. Our study population was heavily transfused at baseline, and therefore, spontaneous improvements in transfusion requirements are thus highly unlikely. The results of the ongoing MDS Event Free Survival with Iron Chelation Therapy Study (TELESTO, clinicaltrials.gov Identifier: NCT00940602) are therefore eagerly anticipated. This will include a composite primary endpoint of death and non-fatal cardiac and hepatic events in lower-risk MDS patients in the setting of a placebocontrolled randomized trial (with secondary outcomes including hematologic and metabolic effects as well as disease progression). This study provides prospective data on which we can build an appropriate evidence base for iron chelation therapy in MDS, which has thus far been a challenge (40). Despite attempts to standardize transfusion practice during the study period, it is acknowledged that transfusion practices may have differed among participating centers before the start of the study. As such, the analysis of erythroid recovery during the trial may be limited. Further confounding of erythroid response analysis may derive from anticipated benefits of deferasirox among physicians leading to a more conservative use of transfusion in a non-blinded setting. Our study lacked imaging data to determine the extent of hepatic and cardiac siderosis, although it should be noted that these tools have not been specifically validated in MDS and serial ferritin measurements provide a good indicator of iron status. Current evidence supports the benefit of deferasirox in lowering liver iron concentration in patients with $\operatorname{MDS}(2,4,5,7$, 8). Although few studies documented myocardial iron overload using $\mathrm{T} 2 *$ magnetic resonance imaging in heavily transfused patients with MDS, others have failed to show significant cardiac siderosis even in patients with severe of hepatic iron overload (41-43). Moreover, reduced overall survival in patients with MDS occurs prior to a transfusion 
history consistent with incident cardiac siderosis and morbidity. This may entail that reductions in labile plasma and nontransferrin-bound iron level such as demonstrated with deferasirox therapy $(3,5)$ may become more relevant considering their early and immediate damaging effects on cardiac myocytes (44-46).

We have demonstrated that deferasirox has an acceptable safety profile and is moderately well tolerated. Deferasirox therapy was associated with improved iron parameters and a concomitant modest reduction in hepatic transaminase concentrations. When taken with the observed hematological responses, these data support the potential of deferasirox to alleviate organ damage and help patients with MDS to achieve transfusion independence.

\section{Acknowledgements}

The authors thank Kaivan Khavandi for medical editorial assistance with this manuscript. Financial support for medical editorial assistance was provided by ASAE-AIL Cagliari. The GIMEMA foundation received an unrestricted grant from Novartis Pharmaceuticals to support trial costs.

\section{Authorship contribution}

E.A., V.C., A.A.DT., G.Q., G.A., M.V., and S.T. provided concept and design; E.A., V.S., A.A.DT., G.Q., C.F., A.V., G.Q., F.R., G.S., D.C., F.S., G.C., A.M., D.V., M.T.V., S.F., L.B., G.L., G.A., and S.S. provided study materials or patients; E.A., V.S., A.A.DT., G.Q., C.F., A.V., G.Q., F.R., G.S., D.C., F.S., G.C., A.M., D.V., M.T.V., S.F., L.B., G.L., G.A., S.S., M.V., and S.T. collected and assembled the data; E.A., A.A.DT., C.F., G.S., D.C., G.A., A.P., P.F., M.V., and S.T. provided data analysis and interpretation; E.A., V.S., and S.T. wrote the manuscript; and all other authors reviewed and contributed their comments to each draft and approved the final version of the manuscript.

\section{Conflicts of interest}

Financial support for medical editorial assistance was provided by ASAE-AIL Cagliari. E.A. has received honoraria from Novartis Pharmaceuticals and is Chair of the Steering Committee of the TELESTO Novartis trial. V.S. has received honoraria from Novartis, Celgene, and Janssen-Cilag. C.F. has received honoraria from Novartis, Celgene, and Janssen-Cilag and research funding from Celgene. M.T.V. has received honoraria from Novartis and Celgene. S.T. has acted as a consultant for Novartis.

\section{Disclaimers}

Dr Angelucci is Chair of the Steering Committee of the TELESTO Novartis trial.

\section{References}

1. Gattermann N, Rachmilewitz EA. Iron overload in MDSpathophysiology, diagnosis, and complications. Ann Hematol 2011;90:1-10.

2. Nolte F, Hochsmann B, Giagounidis A, et al. Results from a 1-year, open-label, single arm, multi-center trial evaluating the efficacy and safety of oral Deferasirox in patients diagnosed with low and int-1 risk myelodysplastic syndrome (MDS) and transfusion-dependent iron overload. Ann Hematol 2013;92:191-8.

3. List AF, Baer MR, Steensma DP, Raza A, Esposito J, Martinez-Lopez N, Paley C, Feigert J, Besa E. Deferasirox reduces serum ferritin and labile plasma iron in RBC transfusion-dependent patients with myelodysplastic syndrome. $J$ Clin Oncol 2012;30:2134-9.

4. Gattermann N, Jarisch A, Schlag R, et al. Deferasirox treatment of iron-overloaded chelation-naive and prechelated patients with myelodysplastic syndromes in medical practice: results from the observational studies eXtend and eXjange. Eur J Haematol 2012;88:260-8.

5. Greenberg PL, Koller CA, Cabantchik ZI, Warsi G, Glynos T, Paley C, Schiffer C. Prospective assessment of effects on iron-overload parameters of deferasirox therapy in patients with myelodysplastic syndromes. Leuk Res 2010;34:1560-5.

6. Gattermann N, Finelli C, Porta MD, et al. Deferasirox in ironoverloaded patients with transfusion-dependent myelodysplastic syndromes: results from the large 1-year EPIC study. Leuk Res 2010;34:1143-50.

7. Metzgeroth G, Dinter D, Schultheis B, Dorn-Beineke A, Lutz K, Leismann O, Hehlmann R, Hastka J. Deferasirox in MDS patients with transfusion-caused iron overload-a phase-II study. Ann Hematol 2009;88:301-10.

8. Porter J, Galanello R, Saglio G, et al. Relative response of patients with myelodysplastic syndromes and other transfusion-dependent anaemias to deferasirox (ICL670): a 1-yr prospective study. Eur J Haematol 2008;80:168-76.

9. Breccia M, Finsinger P, Loglisci G, et al. Deferasirox treatment for myelodysplastic syndromes: "real-life" efficacy and safety in a single-institution patient population. Ann Hematol 2012;91:1345-9.

10. Meerpohl JJ, Antes G, Rucker G, Fleeman N, Motschall E, Niemeyer CM, Bassler D. Deferasirox for managing iron overload in people with myelodysplastic syndrome. Cochrane Database Syst Rev 2010:CD007461.

11. Gattermann N, Finelli C, Della Porta M, et al. Hematologic responses to deferasirox therapy in transfusion-dependent patients with myelodysplastic syndromes. Haematologica 2012;97:1364-71.

12. Greenberg PL, Attar E, Bennett JM, et al. NCCN clinical practice guidelines in oncology: myelodysplastic syndromes. J Natl Compr Canc Netw 2011;9:30-56.

13. Bennett JM. Consensus statement on iron overload in myelodysplastic syndromes. Am J Hematol 2008;83:858-61.

14. Gattermann N. Guidelines on iron chelation therapy in patients with myelodysplastic syndromes and transfusional iron overload. Leuk Res 2007;31(Suppl 3):S10-5. 
15. Hoffbrand AV, Taher A, Cappellini MD. How I treat transfusional iron overload. Blood 2012;120:3657-69.

16. Greenberg P, Cox C, LeBeau MM, et al. International scoring system for evaluating prognosis in myelodysplastic syndromes. Blood 1997;89:2079-88.

17. Oken MM, Creech RH, Tormey DC, Horton J, Davis TE, McFadden ET, Carbone PP. Toxicity and response criteria of the Eastern Cooperative Oncology Group. Am J Clin Oncol 1982;5:649-55.

18. Common Terminology Criteria for Adverse Events (CTCAE), version 3.0. [cited 2013 May 1]. http://ctep.cancer.gov/ protocoldevelopment/electronic_applications/docs/ctcaev3. pdf.

19. Cheson BD, Greenberg PL, Bennett JM, et al. Clinical application and proposal for modification of the International Working Group (IWG) response criteria in myelodysplasia. Blood 2006;108:419-25.

20. Vardiman JW, Thiele J, Arber DA, et al. The 2008 revision of the World Health Organization (WHO) classification of myeloid neoplasms and acute leukemia: rationale and important changes. Blood 2009;114:937-51.

21. Gale RP, Barosi G, Barbui T, et al. What are RBC-transfusiondependence and -independence? Leuk Res 2011;35:8-11.

22. Fine JP, Gray RJ. A proportional hazards model for the subdistribution of a competing risk. J Am Stat Assoc 1999;94:496-509.

23. Linn BS, Linn MW, Gurel L. Cumulative illness rating scale. J Am Geriatr Soc 1968;16:622-6.

24. Charlson ME, Pompei P, Ales KL, MacKenzie CR. A new method of classifying prognostic comorbidity in longitudinal studies: development and validation. J Chronic Dis 1987;40:373-83.

25. Della Porta MG, Malcovati L, Strupp C, et al. Risk stratification based on both disease status and extra-hematologic comorbidities in patients with myelodysplastic syndrome. Haematologica 2011;96:441-9.

26. Cermak J, Kacirkova P, Mikulenkova D, Michalova K. Impact of transfusion dependency on survival in patients with early myelodysplastic syndrome without excess of blasts. Leuk Res 2009;33:1469-74.

27. Malcovati L. Impact of transfusion dependency and secondary iron overload on the survival of patients with myelodysplastic syndromes. Leuk Res 2007;31(Suppl 3):S2-6.

28. Malcovati L, Porta MG, Pascutto C, et al. Prognostic factors and life expectancy in myelodysplastic syndromes classified according to WHO criteria: a basis for clinical decision making. J Clin Oncol 2005;23:7594-603.

29. Rollison DE, Howlader N, Smith MT, Strom SS, Merritt WD, Ries LA, Edwards BK, List AF. Epidemiology of myelodysplastic syndromes and chronic myeloproliferative disorders in the United States, 2001-2004, using data from the NAACCR and SEER programs. Blood 2008;112:45-52.

30. Ghoti H, Fibach E, Merkel D, Perez-Avraham G, Grisariu S, Rachmilewitz EA. Changes in parameters of oxidative stress and free iron biomarkers during treatment with deferasirox in iron-overloaded patients with myelodysplastic syndromes. Haematologica 2010;95:1433-4.

31. Hartmann J, Braulke F, Sinzig U, Wulf G, Maas JH, Konietschke F, Haase D. Iron overload impairs proliferation of erythroid progenitors cells (BFU-E) from patients with myelodysplastic syndromes. Leuk Res 2013;37:327-32.

32. Messa E, Carturan S, Maffe C, et al. Deferasirox is a powerful NF-kappaB inhibitor in myelodysplastic cells and in leukemia cell lines acting independently from cell iron deprivation by chelation and reactive oxygen species scavenging. Haematologica 2010;95:1308-16.

33. Ghoti H, Fibach E, Westerman M, Gordana O, Ganz T, Rachmilewitz EA. Increased serum hepcidin levels during treatment with deferasirox in iron-overloaded patients with myelodysplastic syndrome. Br J Haematol 2011; 153:118-20.

34. Forni GL, Podesta M, Musso M, Piaggio G, Musallam KM, Balocco M, Pozzi S, Rosa A, Frassoni F. Differential effects of the type of iron chelator on the absolute number of hematopoietic peripheral progenitors in patients with betathalassemia major. Haematologica 2013;98:555-9.

35. Takatoku M, Uchiyama T, Okamoto S, et al. Retrospective nationwide survey of Japanese patients with transfusiondependent MDS and aplastic anemia highlights the negative impact of iron overload on morbidity/mortality. Eur $J$ Haematol 2007;78:487-94.

36. Rose C, Brechignac S, Vassilief D, et al. Does iron chelation therapy improve survival in regularly transfused lower risk MDS patients? A multicenter study by the GFM (Groupe Francophone des Myelodysplasies). Leuk Res 2010;34: 864-70.

37. Neukirchen J, Fox F, Kundgen A, Nachtkamp K, Strupp C, Haas R, Germing U, Gattermann N. Improved survival in MDS patients receiving iron chelation therapy - a matched pair analysis of 188 patients from the Dusseldorf MDS registry. Leuk Res 2012;36:1067-70.

38. Nolte F, Angelucci E, Beris P, et al. Clinical management of gastrointestinal disturbances in patients with myelodysplastic syndromes receiving iron chelation treatment with deferasirox. Leuk Res 2011;35:1131-5.

39. Petti MC, Latagliata R, Breccia M, Alimena G, Spadea A, D’Andrea M, Mancini M, Aloe Spiriti MA, Mandelli F. Spontaneous remission in adult patients with de novo myelodysplastic syndrome: a possible event. Haematologica 2001;86:1277-80.

40. Santini V, Alessandrino PE, Angelucci E, et al. Clinical management of myelodysplastic syndromes: update of SIE, SIES, GITMO practice guidelines. Leuk Res 2010;34: 1576-88.

41. Di Tucci AA, Matta G, Deplano S, Gabbas A, Depau C, Derudas D, Caocci G, Agus A, Angelucci E. Myocardial iron overload assessment by $\mathrm{T} 2 *$ magnetic resonance imaging in adult transfusion dependent patients with acquired anemias. Haematologica 2008;93:1385-8.

42. Konen E, Ghoti H, Goitein O, Winder A, Kushnir T, Eshet Y, Rachmilewitz E. No evidence for myocardial iron overload 
in multitransfused patients with myelodysplastic syndrome using cardiac magnetic resonance T2 technique. Am J Hematol 2007;82:1013-6.

43. Pascal L, Beyne-Rauzy O, Brechignac S, et al. Cardiac iron overload assessed by $\mathrm{T} 2 *$ magnetic resonance imaging and cardiac function in regularly transfused myelodysplastic syndrome patients. Br J Haematol 2013;162:413-5.

44. Cabantchik ZI, Breuer W, Zanninelli G, Cianciulli P. LPIlabile plasma iron in iron overload. Best Pract Res Clin Haematol 2005;18:277-87.

45. Breuer W, Ronson A, Slotki IN, Abramov A, Hershko C, Cabantchik ZI. The assessment of serum nontransferrin-bound iron in chelation therapy and iron supplementation. Blood 2000;95:2975-82.

46. Glickstein H, El RB, Link G, Breuer W, Konijn AM, Hershko C, Nick H, Cabantchik ZI. Action of chelators in iron-loaded cardiac cells: Accessibility to intracellular labile iron and functional consequences. Blood 2006;108: 3195-203.

\section{Supporting Information}

Additional Supporting Information may be found in the online version of this article:

Figure S1. Related and non-related adverse events by organ system.

Figure S2. Ferritin values over time after start of deferasirox.

Figure S3. Alanine aminotransferase values over time after start of deferasirox.

Figure S4. Creatinine values over time after start of deferasirox.

Figure S5. Cumulative incidence estimation of transfusion independence in competition with patient death and disease progression.

Table S1. Related grade 3 adverse events.

Table S2. Non-related grade 4-5 adverse events.

Appendix 1. Participating centers and investigators. 